\title{
LUDOLOGIE MÉDIATIQUE
}

Thibault PhilipPetTe ${ }^{1}$ et Maude BonenFanT ${ }^{2}$

Le terme « ludologie » est souvent utilisé comme synonyme de game studies, traduit aussi par « études du jeu » ou « sciences du jeu » en français. D'après Jesper Juul (2011), le terme aurait été employé pour la première fois par Mihály Csíkszentmihályi en 1982 dans un article intitulé Does being human matter? On some interpretive problems of comparative ludology (Ibid., p. 16). Si ce dernier ne donne pas de réelle définition au terme, il part du travail de référence de Johan Huizinga (1938) pour ensuite indiquer que ce qui est intéressant dans le jeu de l'humain, ce ne sont pas tant les caractéristiques de sa conception que la diversité des formes dans lesquelles il se développe, ainsi que des usages qu'il vise.

L'expression connaît un certain intérêt de la part des chercheurs et chercheuses qui étudient les jeux vidéo, principalement suite à l'article de Gonzalo Frasca Ludology meets narratology: similitude and differences between (video)games and narrative (1999). L'auteur y souligne l'importance de ce terme (dérivé du latin ludus) afin de définir cette discipline « en émergence » qui

1 Thibault Philippette est chargé de cours à l'École de communication de l'Université catholique de Louvain, Louvain-la-Neuve (Belgique).

2 Maude Bonenfant est professeure au Département de communication sociale et publique à l'Université du Québec à Montréal (UQAM).

Recherches en communication, n49 - Article publié le 21/03/2019 
cherche à étudier les jeux et les activités de jouer et qui, à l'instar de la narratologie étudiant les récits, serait indépendante du format médiatique sur lequel repose cette activité. Frasca met également de l'avant que la ludologie devrait avoir pour objectif d'étudier les structures et les règles du jeu ainsi que de créer des typologies et des modèles afin de mieux comprendre les mécanismes ludiques (2003). À partir de ce moment, et parallèlement à une croissance impressionnante de l'industrie vidéoludique, ce domaine en pleine explosion voit se multiplier les thématiques de recherche autour du jeu vidéo (Rueff, 2008).

Cependant, l'étude des jeux vidéo n'est certainement pas nouvelle (p. ex. Greenfield, 1984 ; Perriault, 1994) et le mouvement des computer game studies apparu au début des années 2000 (Aarseth, 2001) ne doit pas faire oublier toutes les études d'autres formes plus traditionnelles de jeux, à commencer par celles des jeux de plateau antiques (Finkel, 1997) ou celles des animaux dès la fin du XIX ${ }^{\mathrm{e}}$ siècle (Groos, 1896). D'autres formes d'études, dans d'autres contextes, ont également contribué, d'une certaine manière, au développement de la ludologie, que ce soit en ethnographie (Culin, 1907), en psychologie (Piaget, 1945), en sociologie (Mead, 1934 ; Caillois, 1958), en communication (Bateson, 1972), en éducation (Sutton-Smith, 1979), en histoire (Murray, 1913 ; Bell, 1960), etc. Même les mathématiques ont exploré la théorie des jeux (Von Neumann et Morgenstern, 1944) et la philosophie s'est intéressée aux jeux de langage (Wittgenstein, 1953) ou au jeu existentiel (Fink, 1960 ; Axelos, 1969).

En fait, historiquement, la ludologie concerne une panoplie de facettes du jeu et de l'activité ludique, et vise à mieux comprendre l'expression de ce phénomène. Alors que certains notent une décroissance de la « substance ludique » des sociétés modernes (Huizinga, 1938 ; Caillois, 1958), d'autres remettent en question cette vision (Henriot, 1969 ; Cotta, 1980). En effet, Henriot (1989) relève l'importance de plus en plus croissante de cette idée de jeu dans nos sociétés contemporaines. Minnema (1998) renchérit en expliquant les raisons de la montée des études du jeu dans notre société postmoderne. Il démontre à quel point nous nous reconnaissons dans le phénomène ludique autant que dans la méta- 
phore : le jeu fait écho à qui nous sommes et au monde dans lequel nous évoluons.

Ainsi, après l'explosion des études vidéoludiques au début des années 2000, la ludologie fait désormais un retour à ses origines, surtout depuis les années 2010, et explore à nouveau toutes formes de jeux et de jouer et ce, sous divers supports médiatiques : jeux à vocation sociale (McGonigal, 2011), ludification (Raessens, 2014), jeux de rôles (Kapp, 2015 ; Zagal et Deterding, 2018), jeux télévisés (Leveneur, 2006), jeux esthétiques (Flanagan, 2014), etc. En fait, Genvo affirme que « la ludologie doit être définie comme la discipline qui étudie le jeu, dans la signification la plus large de ce terme [... il s'agit] de prendre en considération l'ensemble des analyses qui se portent sur les phénomènes ludiques en général. Et dans ce cadre, la ludologie se doit d'être interdisciplinaire » (Genvo, 2008, p. 100). Solinski prolonge son idée en indiquant que la ludologie est « un langage commun à même d'enrichir les sciences humaines et sociales en rassemblant, articulant et créant des principes de compréhension du ludique »(Solinski, 2015, p. 16).

Partant de ce cadrage général et de ce positionnement, le parti pris de cet appel de Recherches en Communication a été d'offrir un espace permettant d'interroger la place du ludique dans la sphère plus large des pratiques médiatiques contemporaines. De la sorte, nous voulions à la fois intéresser des auteur $\cdot e \cdot s$ ne se revendiquant pas forcément des études du jeu ou étudiant spécifiquement des objets-jeux, et à la fois traiter de formats médiatiques qui, sans pouvoir être actuellement considérés pleinement comme des jeux, semblaient de plus en plus s'en inspirer. Afin d'ouvrir largement le champ des possibles, tout en l'inscrivant dans celui des études médiatiques, l'appel s'est construit à partir de la définition de «média » proposée par James Anderson (1988, p. 11), à savoir " une activité humaine distincte qui organise la réalité en textes lisibles en vue de l'action $»$. Si tout média ne possède pas toutes les composantes proprement ludiques (p. ex. « second degré », « incertitude », « suite de décisions », « règle », « frivolité ». Cf. Brougère, 2005), cette définition permet d'interroger les éventuelles dimensions ludiques d'un média, autant du point de 
vue de sa matérialité (le texte lisible) que d'un point de vue plus social (l'activité qu'il sous-tend).

Dans cet appel à communications, nous avons ainsi identifié cinq axes non exclusifs autour desquels les propositions pouvaient être construites.

Le premier axe s'intéressait à la conception, c'est-à-dire à comment conçoit-on des dispositifs ou objets médiatiques en incluant des mécanismes de jeu ? Avec quelles intentions et objectifs ? Comment générer une « idée de jeu » dans l'esprit des participant·e·s ? L'appel ouvrait à une grande diversité de formes médiatiques (parcours muséaux, reportages photo immersifs, films d'animation, etc.).

Au travers du deuxième axe, nous souhaitions voir traiter la question des pratiques. Comment certains contextes ont vu émerger des pratiques considérées aujourd'hui comme des formes de jeux (musique, théâtre, cinéma) et comment cela semble apparaître plus récemment dans de nouveaux contextes (organisations, écoles, hôpitaux, etc.). Il s'agissait à la fois d'interroger ces transformations « ludiques », mais aussi leurs raisons d'être : qu'apporte l'usage du jeu ou des jeux ? Quels en sont les freins et facilitateurs? Quelles en sont les parties prenantes et pourquoi?

Le troisième axe portait sur les métiers en interrogeant la création potentielle de nouveaux métiers, ou à tout le moins la convergence de différents métiers derrière ces productions, que l'on songe à la rencontre de chercheurs, chercheuses en jeu et d'artistes pour la création d'œuvres interactives ludiques dans les espaces publics, de spécialistes de la narration et de l'interactivité dans la production de webdocumentaires, de technicien'ne's de l'animation et de metteurs, metteuses en scène dans certaines productions théâtrales, d'enseignant·e·s et de concepteurs, conceptrices de jeux dans la réalisation de jeux sérieux, etc.

Le quatrième axe proposait également d'interroger le statut de la recherche en sciences du jeu par rapport aux études en communication et aux analyses médiatiques. En effet, la genèse des recherches en communication est celle d'une interdiscipline, influencée, sans être exhaustif, par des travaux en ingénierie de télécommunications, en linguistique structurale, en études poli- 
tiques des effets des médias de masse, en psychologie sociale, en sémiotique et pragmatique, en narratologie, en sociologie interactionniste ou encore par des approches plus systémiques des organisations humaines. Cette ouverture du champ est une faiblesse de par la multiplicité des modèles et concepts rendant parfois certaines perspectives inconciliables (Meunier \& Peraya, 1993, p. 28), mais une force en ce que cette souplesse disciplinaire permet des ajustements théoriques et méthodologiques aux évolutions des médias (au sens précité). Ce même constat d'ouverture ayant été posé à la ludologie ou aux études des jeux (cf. supra), cet axe proposait dès lors d'interroger les spécificités et les zones de confluence entre ludologie et recherches médiatiques.

Enfin le dernier axe invitait à proposer une réflexion plus politique et éthique sur cette place que l'on veut donner au jeu dans nos sociétés postmodernes. Vouloir « tout jouer » ne tuet-il pas le fondement même du jeu ? Ces tendances ne sont-elles pas dictées par des acteurs cherchant à pénétrer de nouveaux marchés économiques ? Lorsque le jeu n'est plus simplement conçu comme une forme de divertissement, mais comme un attracteur politique voire existentiel, comment prévenir des effets idéologiques recherchés et éduquer à pouvoir déconstruire ces messages et intentions en adoptant une posture plus critique (Bonenfant et Philippette, 2018).

Nous espérons que ce numéro rencontrera vos intérêts et questionnements. Nous vous en souhaitons en tous les cas une excellente lecture.

\section{Références}

Aarseth, E. (2001). Computer Game Studies, Year One. The International Journal of Computer Game Research 1(1). Disponible à : http://gamestudies.org/0101/editorial.html.

Anderson, J. A. (1988). Examen de quelques concepts éclairant la position de l'éducateur aux médias. In Actes du Symposium de Lausanne (Vol. 23, pp. 11-23). Lausanne : Centre d'initiation aux communications de masse.

Axelos, K. (1969). Le jeu du monde. Coll. Argument. Paris : Éditions de Minuit.

Bateson, G. (1972). Steps to an ecology of mind. Chicago : University of Chicago Press.

Bell, R. C. (1960). Board and Table Games from many civilizations. London : Oxford University Press. 
Bonenfant, M. \& Philippette, T. (2018). Rhétorique de l'engagement ludique dans des dispositifs de ludification. Sciences $d u$ Jeu, 10. Disponible à : https://journals.openedition.org/sdj/1422.

Brougère, G. (2005). Jouer/Apprendre. Paris : Economica.

Caillois, R. (1967 [1958]). Les jeux et les hommes (Le masque et le Vertige). Coll. Folio essais. Paris : Gallimard.

Cotta, A. (1980). La société ludique : la vie envahie par le jeu. Paris : Grasset.

Csíkszentmihályi, M. (1982). Does being human matter? On some interpretive problems of comparative ludology. Dans P.K. Smith (Éd.), Does play matter? Functional and evolutionary aspects of animal and human play, The behavioral and Brain Sciences. (p. 160). Cambridge : Cambridge University Press.

Culin, S. (1986 [1907]). Games of the North American Indians. New York : Dover.

Fink, E. (1965 [1960]). Le jeu comme symbole du monde. Coll. Arguments. Paris : Éditions de Minuit.

Finkel, I. (1997). Ancient Board Games. New York : Stewart Tabori \& Chang.

Flanagan, M. (2014). Playful Aesthetics : Toward a ludic language. Dans S. P. Waltz \& S. Deterding (Éd.), The Gameful World: Approaches, issues, Applications (pp. 249273). Cambridge MA : The MIT Press.

Frasca, G. (1999). Ludology meets Narratology: similitude and differences between (video)games and narrative. Parnasso, 3. Disponible à : https://www.ludology.org/ articles/ludology.htm.

Frasca, G. (2003). Simulation versus narrative : introduction to ludology. Dans J.P. Wolf et B. Perron (Éd.), The Video Game Theory Reader (pp. 221-236). New York : Routledge.

Genvo, S. (2008). Réflexions ludologiques. Médiamorphoses 22, 95-101.

Greenfield, P.M. (1984). Mind and Media: The effects of Television, Video Games and Computers (Third Printing). London : Harvard University Press.

Groos, K. (1898 [1896]). The play of Animals. New York : D. Appleton.

Henriot, J. (1969). Le Jeu. Paris : Presses universitaires de France.

Henriot, J. (1989). Sous couleur de jouer. Paris : José Corti.

Huizinga, J. (2008 [1938]). Homo Ludens : essai sur la fonction sociale du jeu. Paris : Gallimard.

Juul, J. (2011). Half-Real: Video Games Between Real Rules and Fictional Worlds. Cambridge MA : The MIT Press.

Kapp, S. (2015). Quitter le donjon. Quand le jeu de rôles devient « grandeur nature ». Sciences du Jeu, 4. Disponible à : https://journals.openedition.org/sdj/488.

Leveneur, L. (2006). « Du télé-viseur au téléspectateur ». Questions de communication $10,333-348$.

McGonigal, J. (2011). Reality Is Broken: Why Games Make Us Better and How They Can Change the World. New York : Penguin Random House.

Mead, G. H. (1934). Mind, Self, and Society. Chicago : University of Chicago Press.

Meunier, J.-P. \& Peraya, D. (1993). Introduction aux Théories de la Communication. Bruxelles : De Boeck Université.

Minnema, L. (1998). Play and (post)modern culture: Essay on changes in the scientific interest in the phenomenon of play. Cultural Dynamics 10 (1), 21-47.

Murray, H. J. R. (1913). History of Chess. London : Oxford University Press. 
Perriault, J. (1994). L'acquisition et la construction de connaissances par les jeux informatisés. Réseaux 12(67), 57-70.

Piaget, J. (1945). La formation du symbole chez l'enfant : imitation, jeu et rêve, image et représentation. Paris : Delachaux et Niestlé.

Raessens, J. (2014). The Ludification of Culture. Dans M. Fuchs, S. Fizek, P. Ruffino \& N. Schrape (Éd.), Rethinking Gamification (pp. 91-114). Lüneburg : Meson Press.

Rueff, J. (2008). Où en sont les « game studies » ? Réseaux 151(5), 139-166.

Solinski, B. (2015). Ludologie : jeu, discours, complexité. Dissertation doctorale, Université de Lorraine. Disponible à : https://hal.univ-lorraine.fr/tel-01751890/document.

Sutton-Smith, B. (1979). Play and learning. New York : Gardner Press.

Von Neumann, J. \& Morgenstern, O. (1944). Theory of Games and Economic Behavior. Princeton : Princeton University Press.

Wittgenstein, L. (1961 [1953]). Investigations philosophiques. Paris : Gallimard.

Zagal, J. P. \& Deterding, S. (2018). Role-Playing Game Studies. New York : Routledge. 


\section{(C) $(9) \bigodot_{\text {BY NC }}$ Publié sous la licence Creative Commons}

«Attribution - Pas d'Utilisation Commerciale - Pas de Modification 4.0 International» (CC BY-NC-ND) 